\title{
Elevated CD44 expression predicts poor prognosis in patients with low-grade glioma
}

\author{
QIANG DONG ${ }^{1 *}$, QIAO LI ${ }^{1 *}$, MAOLIN WANG $^{1}$, JIANHONG HU ${ }^{1}$, JUNQIANG DAI ${ }^{1}$, \\ LIANG NIU $^{1}$, GUOQIANG YUAN ${ }^{2}$ and YAWEN PAN ${ }^{1,2}$ \\ ${ }^{1}$ Department of Neurosurgery, Lanzhou University Second Hospital; \\ ${ }^{2}$ Institute of Neurology, Lanzhou University, Lanzhou, Gansu 730030, P.R. China
}

Received November 23, 2018; Accepted June 20, 2019

DOI: $10.3892 / \mathrm{ol} .2019 .10728$

\begin{abstract}
CD44 is involved in malignant processes including cell motility, tumor growth and angiogenesis. To explore the potential role of CD44 as a prognostic biomarker in low grade gliomas (LGG), the mRNA expression levels of CD44 in tissues from 12 patients with glioma were evaluated by microarray analysis. The mRNA level of CD44 in LGG and glioblastoma multiforme (GBM) were analyzed using datasets downloaded from the publicly available Oncomine database. Reverse transcription-quantitative PCR and western blotting were used to further analyze the CD44 expression level in a set of 53 patients. Kaplan-Meier analysis was performed to identify the prognostic roles of CD44 mRNA in LGG and GBM, with data obtained from the OncoLnc and Gene Expression Profiling Interactive Analysis databases and clinical follow-ups. The present results revealed that CD44 mRNA expression levels were elevated in LGG and GBM compared with normal brain tissues. Furthermore, increased CD44 expression was associated with poor survival rates in LGG. The present study suggested that CD44 may act as an independent prognostic factor for LGG, and may be a potential therapeutic target for gliomas.
\end{abstract}

\section{Introduction}

Gliomas are the most common malignant primary brain tumors, and are categorized, according to the 2016 World

Correspondence to: Professor Yawen Pan, Department of Neurosurgery, Lanzhou University Second Hospital, 82 Cuiyingmen Road, Chengguan, Lanzhou, Gansu 730030, P.R. China

E-mail: panyw2018@163.com

Dr Guoqiang Yuan, Institute of Neurology, Lanzhou University Second Hospital, 82 Cuiyingmen Road, Lanzhou, Gansu 730030, P.R. China E-mail: 13893292173@163.com

${ }^{*}$ Contributed equally

Key words: CD44, glioma, prognosis, bioinformatics analysis
Health Organization (WHO) classification, as low-grade (grade I-II) and high-grade (grade III-IV) (1). Low-grade gliomas (LGGs) are infiltrative neoplasms that most frequently arise in the cerebral hemispheres of adults, and include astrocytomas, oligodendrogliomas and oligoastrocytomas (1). LGGs generally occur in young adults between 35 and 44 years of age (2). Despite a better prognosis compared with HGG, complete neurosurgical resection of LGGs is challenging due to their invasive nature, increased vascularity and lack of a well-defined tumor capsule. Furthermore, certain cases LGG may rapidly progress to WHO grade IV glioma, becoming a glioblastoma multiforme (GBM), whereas others may remain stable for a long time (3). The survival time of patients with LGG ranges from 1 to 15 years (4).

Although traditional histopathological methods are regarded as the gold standard for LGG classification, they do not adequately predict clinical outcome. Consequently, an increasing number of studies have shown that biomarkers may improve the clinical decision-making process (5). Hypermethylation of the $\mathrm{O}^{6}$-methylguanine-DNA methyltransferase promoter can effectively predict the responsiveness to temozolomide (6). Mutations in isocitrate dehydrogenase $\left(\mathrm{NADP}^{+}\right) 1$ (IDH1) and IDH2 may result in an improved prognosis for patients with LGG. Mutations in IDH and the heterozygous deletion at the chromosomal position $1 \mathrm{p} / 19 \mathrm{q}$ may result in improved responses to radiotherapy and chemotherapy, and patients with these mutations have longer survival times than patients without these mutations (7-10).

The present study investigated novel prognostic and predictive biomarkers for LGG using an mRNA PCR array to screen for genes with altered expression in LGG. The prognostic value of newly identified biomarkers was subsequently investigated using the OncoLnc and Gene Expression Profiling Interactive Analysis (GEPIA) databases, and biomarker mRNA level was analyzed using the Oncomine database.

\section{Materials and methods}

Patient specimens. The study included 53 glioma tissues and formalin-fixed paraffin-embedded tumortissues.Gliomatissues were fixed overnight at $4^{\circ} \mathrm{C}$ in $10 \%$ neutral-buffered formalin, dehydrated by soaking in $70,85,95$ and $100 \%$ ethanol for $45 \mathrm{~min}$ each time at room temperature, washed in xylene 
twice for $15 \mathrm{~min}$ each at room temperature, soaked twice in paraffin for $1 \mathrm{~h}$ each at $56^{\circ} \mathrm{C}$, embedded in paraffin and subsequently stored at $4^{\circ} \mathrm{C}$ until use. Six patients with LGG and six patients with GBM were recruited from the Neurology Institute of Lanzhou University Second Hospital (Lanzhou, China) between January 2013 and December 2017. Of the patients recruited in the present study, 31 were male and 22 were female, with a mean age of 48 years (range, 20-72 years). A total of five normal brain tissue samples were obtained from patients without glioma who underwent surgery for other reasons, including cerebral trauma. The glioma and normal tissue specimens were snap-frozen in liquid nitrogen following surgery, and subsequently stored at $-80^{\circ} \mathrm{C}$ until use. Histological diagnosis was based on the criteria stated by the WHO. According to the 2016 World Health Organization Classification of Tumors of the Central Nervous System (1), gliomas were divided into four categories: i) WHO I (pilocytic astrocytoma); ii) WHO II (diffuse astrocytoma, oligoastrocytoma and oligodendroglioma); iii) WHO III (anaplastic astrocytoma, anaplastic oligodendroglioma and anaplastic oligoastrocytoma); and iv) WHO IV (glioblastoma). Patients undergoing surgery who had not been previously treated with radiotherapy or chemotherapy were included in the present study. Approval for the study was obtained from The Medical Ethics Committee of The Affiliated Second Hospital of Lanzhou University. Written informed consent was obtained from all recruited patients.

Human mRNA PCR array. After dewaxing, total RNA was extracted from the tumor samples of 12 randomly selected patients using TRIzol ${ }^{\circledR}$ reagent (Thermo Fisher Scientific, Inc.). First strand cDNA was synthesized using the mRNA First-Strand cDNA Synthesis kit (cat. no. AS-MR-004; Arraystar, Inc.) according to the manufacturer's protocol. The cDNA products were amplified using TB SYBR green Premix Ex Taq II (Takara Biotechnology Co., Ltd.). The Human mRNA PCR Array (cat. no. AS-MR-0033, Arraystar, Inc.) was used with the ABI PRISM7900 system (Applied Biosystems; Thermo Fisher Scientific, Inc.). The thermocycling condition of the qPCR were: Initial denaturation at $95^{\circ} \mathrm{C}$ for $1 \mathrm{~min}$, followed by 40 cycles of denaturation at $95^{\circ} \mathrm{C}$ for $15 \mathrm{sec}$, and annealing and extension at $60^{\circ} \mathrm{C}$ for $30 \mathrm{sec}$. Each 364 -well miRStar Human Cancer Focus miRNA and Target mRNA PCR Array (Arraystar, Inc.) contains 177 genes associated with human cancer, six wells for housekeeping genes, a genomic DNA contamination control, three replicates of reverse transcription (RT) controls and three replicates of positive PCR controls. The raw data were processed by performing the following analyses: Background detection, robust multi-array average global background correlation, quantile normalization, median adjustment and log2-transformation with miRNA QC tool version 1.1 (Affymetrix; Thermo Fisher Scientific, Inc.).

Oncomine database analysis. The expression level of CD44 in LGG, GBM and matched normal tissues were retrieved from the Oncomine database (http://www.oncomine.org). The expression analysis of CD44 in the Oncomine database was based on a previous study (11). A total of 157 brain and central nervous system tumors and 23 normal brain samples were analyzed on an Affymetrix U133 Plus 2.0 platform (Affymetrix; Thermo
Fisher Scientific, Inc.). Normal tissue samples were provided in the Oncomine database. The-fold-change of mRNA expression in LGG and GBM samples, compared with their matched normal tissues, was determined using the following parameters: i) $\mathrm{P}<1 \times 10^{-3}$; and ii) fold-change $\geq 2$-fold.

Kaplan-Meier plotter analysis. The prognostic value of CD44 was analyzed using OncoLnc (www.oncolnc.org/search_ results/?q=CD44) and GEPIA (gepia.cancer-pku.cn). Patients were divided into high-level and low-level groups based on the median value of CD44. The overall survival (OS) rates of the patients in the high-level and low-level CD44 groups were evaluated using Kaplan-Meier analysis (gepia.cancer-pku. $\mathrm{cn} /$ detail.php?gene $=\&$ clicktag=survival $)$.

$R T$-quantitative PCR (RT-q) PCR. Total RNA was extracted from normal brain and glioma tissues using Trizol ${ }^{\circledR}$ reagent (Thermo Fisher Scientific, Inc.) according to the manufacturer's protocol. Total RNA ( $2 \mu \mathrm{g})$ was reverse transcribed using the PrimeScript RT reagent kit with genomic DNA Eraser (Takara Biotechnology Co., Ltd.). Reverse transcription was performed as follows: $37^{\circ} \mathrm{C}$ for $15 \mathrm{~min}$ and $85^{\circ} \mathrm{C}$ for $5 \mathrm{sec}$. The cDNA products were amplified using TB SYBR green Premix Ex Taq II (Takara Biotechnology Co., Ltd.) on a Bio-Rad CFX96 real-time PCR system (Bio-Rad Laboratories, Inc.). The qPCR procedure was performed as follows: Pre-denaturation at $95^{\circ} \mathrm{C}$ for $1 \mathrm{~min}$, followed by 40 cycles of denaturation at $95^{\circ} \mathrm{C}$ for $15 \mathrm{sec}$, and annealing and extension at $60^{\circ} \mathrm{C}$ for $30 \mathrm{sec}$. The relative mRNA expression data were analyzed using the $2^{-\Delta \Delta \mathrm{Cq}}$ method (10), and expression values were normalized to the internal control GAPDH. The following primers were used: CD44 forward, 5'-CCAACTCCATCTGTGCAG-3' and reverse, 5'-AACCTCCTGAAGTGCTGC-3'; GAPDH, forward 5'-GGACCTGACCTGCCGTCTAG-3' and reverse, 5'-TAG CCCAGGAGGATGCCCTTGAG-3'.

Western blotting. The glioma tissues were homogenized and lysed with cell lysis buffer (Beijing Solarbio Science \& Technology Co., Ltd.) containing 1\% PMSF. The lysates were centrifuged at $12,000 \mathrm{x} \mathrm{g}$ for $5 \mathrm{~min}$ at $4^{\circ} \mathrm{C}$. The protein concentration was determined using a bicinchoninic acid assay. The proteins (30 $\mu \mathrm{g}$ total protein/lane) were separated using SDS-PAGE on a $12 \%$ gel. Proteins were transferred to PVDF membranes (EMD Millipore), blocked with 5\% fat-free milk for $25^{\circ} \mathrm{C}$ for $1 \mathrm{~h}$ and incubated with primary antibodies against CD44 (cat. no. D190741; 1:750; Sangon Biotech Co., Ltd.) and GAPDH (cat. no. TA-08; 1:1,000; Beijing Zhongshan Jinqiao Biotechnology Co., Ltd.) at $4^{\circ} \mathrm{C}$ overnight. The membranes were washed with PBS and $0.1 \%$ Tween, and incubated with goat anti-mouse secondary antibody (cat. no. abs20001; 1:2,000; Absin Bioscience Inc.) at $25^{\circ} \mathrm{C}$ for $1 \mathrm{~h}$. Protein bands were visualized using the enhanced chemiluminescence (PerkinElmer, Inc.) method and a Bio-Rad gel imaging system (Bio-Rad Laboratories, Inc.), and densitometry analysis was performed using Image J version 1.8.0 software (National Institutes of Health).

Data analysis. The median mRNA expression levels of CD44 mRNA was used as the cut-off point, and the patients were divided into a high-level and a low-level group. The $\chi^{2}$ test was 
used to analyze the association between CD44 mRNA expression and clinicopathological characteristics. The OS rates of LGG in the high- and low-level groups were evaluated using Kaplan-Meier analysis. Furthermore, univariate and multivariate Cox proportional hazard regression analysis was performed to evaluate the prognostic value of multiple variables, including CD44 mRNA expression, sex, age and Karnofsky Performance Scale (KPS) (12).

Statistical analysis. All analyses were performed using SPSS software (version 17; SPSS, Inc.). The data are presented as the mean \pm SEM. Data were analyzed using one-way ANOVA followed by the least significant difference post hoc test or an unpaired Student's t-test. Survival curves were estimated using the Kaplan-Meier method with the log-rank test. Univariate and multivariate Cox regression analysis was employed to estimate the risk factor of gliomas. $\mathrm{P}<0.05$ was considered to indicate a statistically significant difference. The analyses were performed using GraphPad Prism software (version 5; GraphPad Software, Inc.).

\section{Results}

Gene expression analysis. The mRNA profile was analyzed for LGG $(n=6)$ and GBM $(n=6)$ using the miRStar Human Cancer Focus mRNA PCR Array. The median expression levels of each mRNA were calculated in both groups, and the differences between them were determined using a t-test. $\mathrm{P}<0.05$ was considered to indicate a significant difference and a-fold change $>2$ was used as the cut-off value. The results indicated that of the 177 genes, 44 difference genes were upregulated. CD44 was identified to exhibit a 3-fold increase in GBM ( $\mathrm{P}=0.02$; Fig. 1). The results were further validated using Oncomine analysis, which demonstrated that the CD44 mRNA level was significantly higher in GBM compared with LGG and normal brain tissue (Fig. 2A and B).

CD44 expression in glioma tissues. To validate the mRNA PCR array and Oncomine database findings, the expression level of CD44 was determined in the glioma tissue samples $(\mathrm{n}=53)$ and normal brain tissues $(\mathrm{n}=5)$ using RT-qPCR and western blotting. The CD44 expression level in glioma tissues was significantly increased compared with normal brain tissues $(\mathrm{P}<0.05$; Fig. $3 \mathrm{~A}$ and $\mathrm{B})$. The data were consistent with the results of the mRNA PCR array and Oncomine database analysis, in which patients were divided into high-level $(\mathrm{n}=26)$ and low-level groups $(n=27)$, with the median expression level of CD44 mRNA as the cut-off value. The expression level of CD44 mRNA was associated with KPS $(\mathrm{P}<0.01)$ and WHO grade $(\mathrm{P}<0.01$; Table I). However, it was not significantly associated with other clinicopathological parameters, including age and sex.

Survival analysis. Prognostic value analysis of CD44 mRNA was performed in patients with LGG and GBM using data from the OncoLnc and GEPIA databases. Kaplan-Meier analysis revealed that a high expression level of CD44 in LGG was a predictor of short $\mathrm{OS}$ and poor patient outcome, as assessed by OncoLnc $(\mathrm{P}<0.01)$ and GEPIA $(\mathrm{P}<0.01)$ (Fig. 4A and B). The level of CD44 mRNA did not significantly influence patient

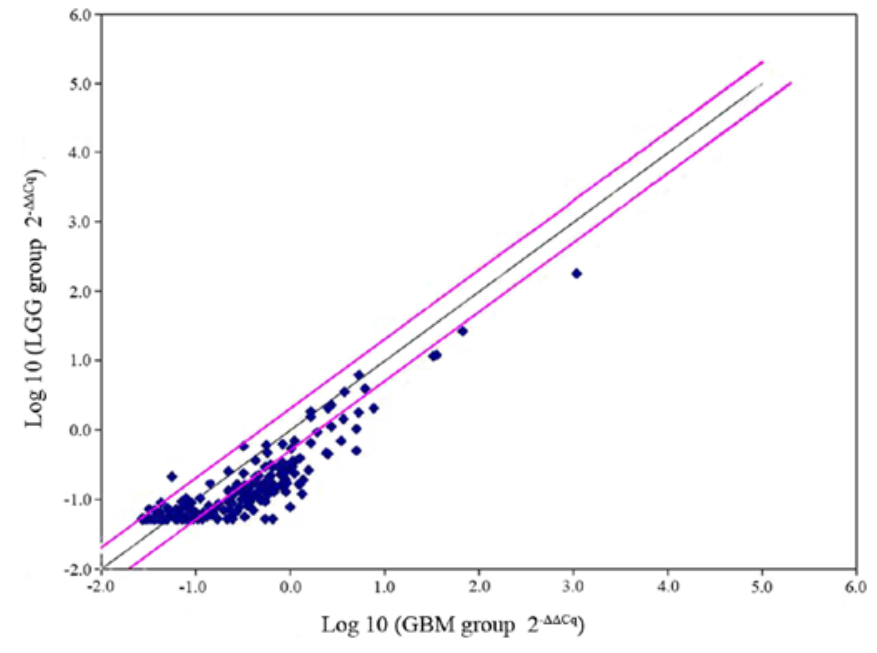

Figure 1. Screening of 177 cancer-associated genes by miRStar Human Cancer Focus miRNA and Target mRNA PCR array. The results were analyzed using a PCR array ( $\mathrm{n}=6$ samples per group). A total of 44 genes were identified with $>2$-fold change between LGG and GBM. LGG, low-grade glioma; GBM, glioblastoma multiforme.

outcome, as assessed by OncoLnc $(\mathrm{P}=0.099)$ and GEPIA $(\mathrm{P}=0.052)$ in GBM (Fig. 4D and E). In addition, Kaplan-Meier analysis and the log-rank test were used to evaluate the prognostic value of CD44 mRNA expression in patients with LGG and GBM according to clinical follow-up data. The present results suggested that patients with LGG with a high expression level of CD44 had a significantly shorter OS than the low expression group ( $\mathrm{P}=0.035$; Fig. $4 \mathrm{C})$. There was no significant difference in patients with GBM ( $\mathrm{P}=0.123$; Fig. 4F). Furthermore, Cox regression analysis revealed that the expression level of CD44 was significantly associated with the OS of LGG patients (hazard ratio, 3.7012; 95\% CI, 0.927-11.215; $\mathrm{P}=0.032$; Table II).

\section{Discussion}

Despite recent progresses in neurosurgery, the survival rate of patients with LGG varies widely. To improve prediction accuracy for LGG, molecular markers such as ATRX chromatin remodeler, $\mathrm{p} 53$ and telomerase reverse transcriptase have been established $(13,14)$. Furthermore, IDH mutation and 1p/19q codeletion were used in the 2016 WHO classification of central nervous system tumors (1). IDH-wild-type and IDH-mutants are observed in 90 and 10\%, respectively, of all GBM cases (15). Present studies for the classification of glioma are focused on high-grade glioma; therefore, biomarkers relevant for the prognostic stratification of patients with LGG remain limited.

In recent years, there have been rapid developments in molecular biology techniques, including chromatin immunoprecipitation and high-throughput sequencing, as well as in the application of bioinformatics methods. Advances in these techniques have allowed the investigation of the occurrence and development of glioma at the molecular level (16-18). The present study used a PCR array, and identified CD44 expression level to be associated with the histopathological grade of gliomas. Western blotting suggested that CD44 expression was higher 

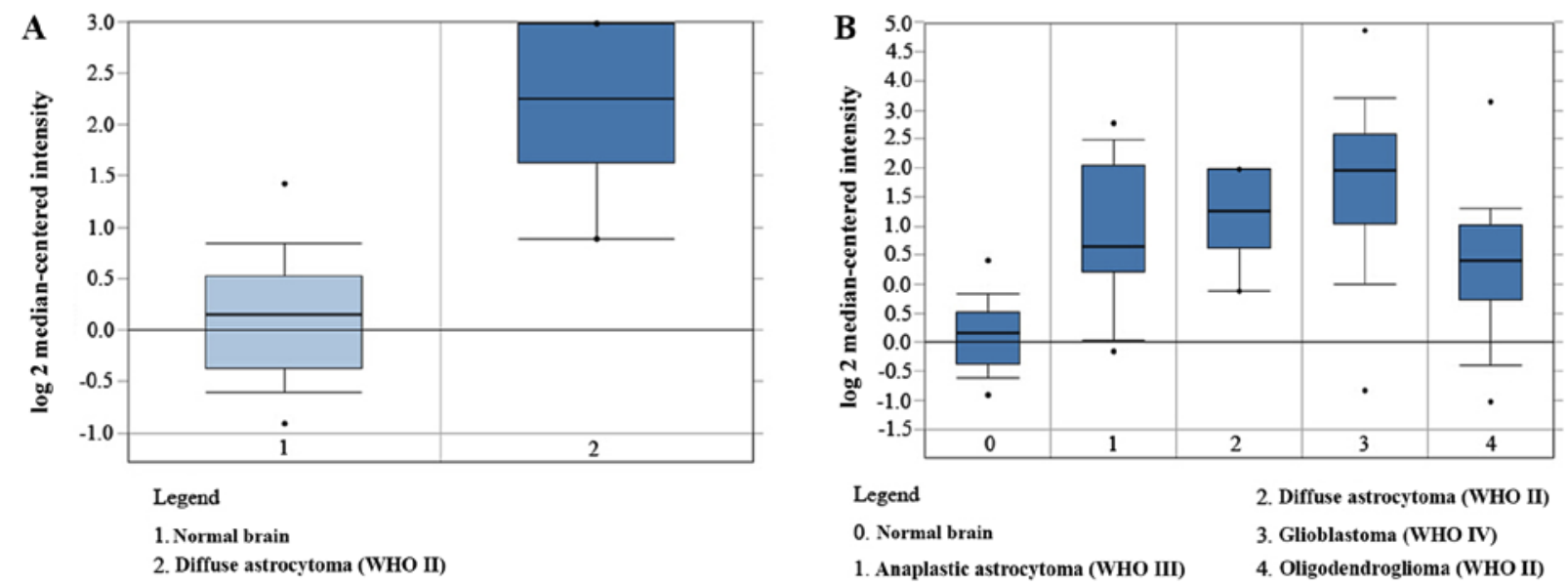

Figure 2. CD44 mRNA expression level was evaluated using Oncomine analysis. (A) CD44 mRNA expression in low-grade glioma. (B) CD44 mRNA expression in different grades of gliomas. Normal brain tissues, WHO II (including diffuse astrocytoma and oligodendroglioma), WHO III (anaplastic astrocytoma) and WHO IV (glioblastoma). WHO, World Health Organization.
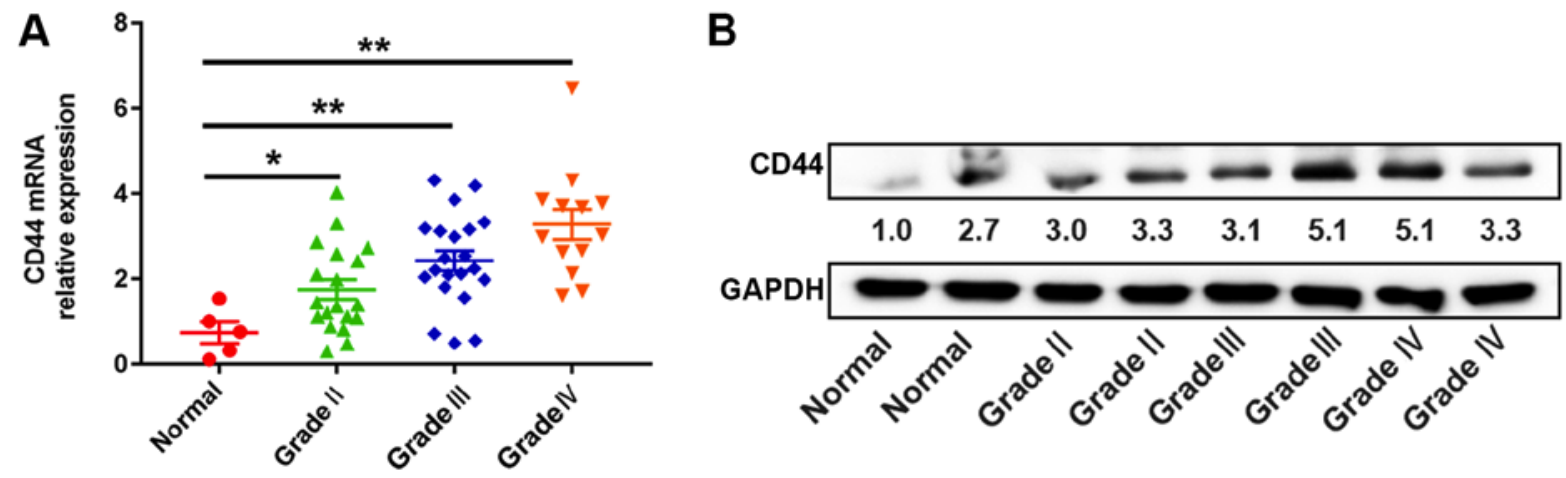

Figure 3. Expression levels of CD44 in glioma tissues. (A) Expression patterns of CD44 in different grade glioma tissues were analyzed by reverse-transcription quantitative PCR. (B) The protein expression levels of CD44 in patients with different grade glioma tissues were analyzed by western blotting. The numbers under the blot represents the protein expression levels of CD44 in glioma tissues of different grades relative to the respective GAPDH loading controls. "P $<0.05$, ${ }^{* * *} \mathrm{P}<0.01$.
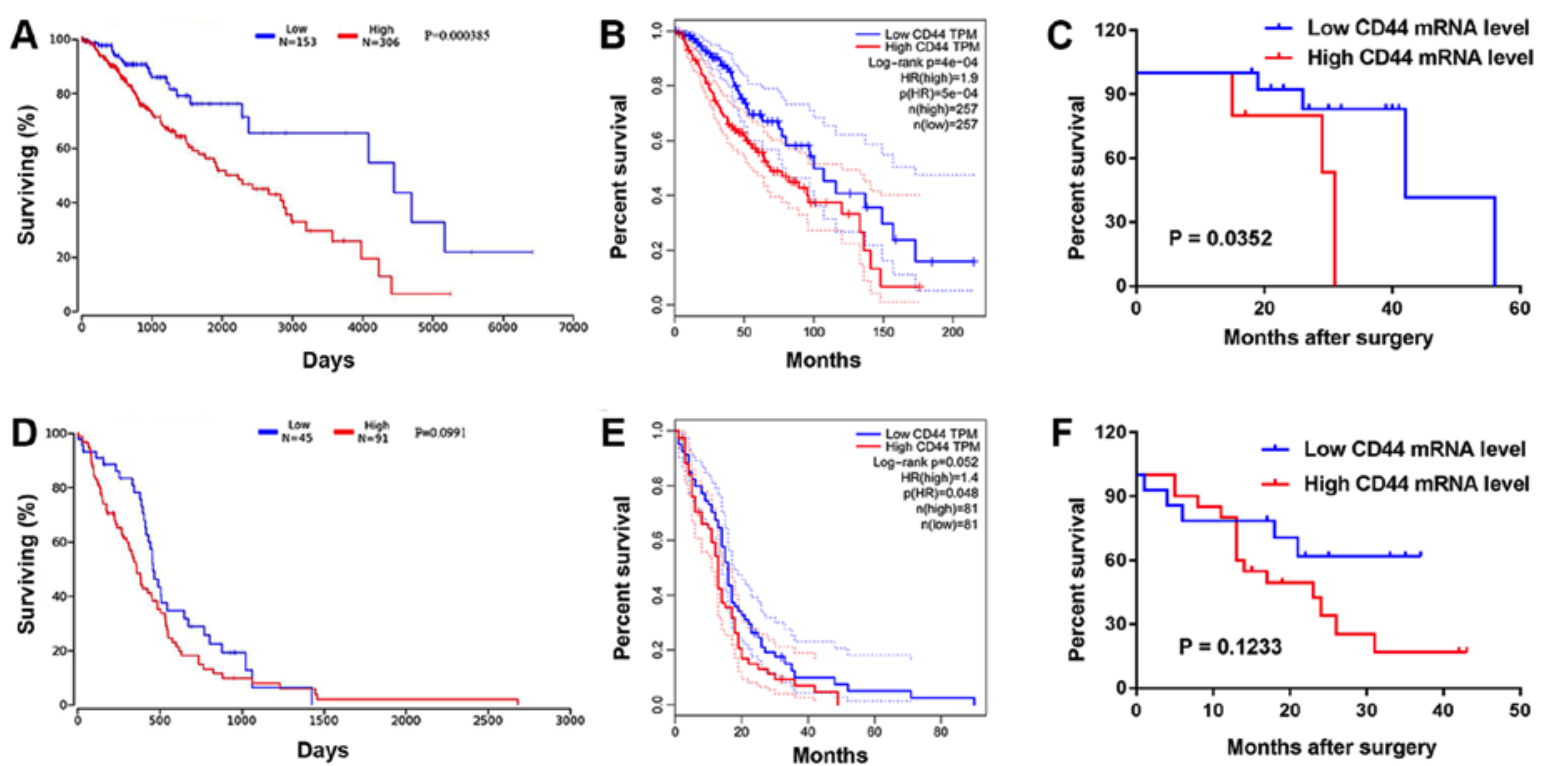

Figure 4. Kaplan-Meier curves for OS in patients with gliomas. (A) Prognostic significance of CD44 was analyzed in LGG using the OncoLnc databases. (B) Prognostic significance of CD44 was analyzed in LGG using the GEPIA databases. (C) Analysis of survival curves in patients with LGGs by Kaplan-Meier survival analysis. (D) The prognostic significance of CD44 was analyzed in GBM by OncoLnc databases. (E) The prognostic significance of CD44 was analyzed in GBM by GEPIA databases. (F) Analysis of survival curves in patients with GBM by Kaplan-Meier survival analysis. OS, overall survival; LGG, low-grade glioma; GEPIA, Gene Expression Profiling Interactive Analysis; GBM, glioblastoma multiforme; HR, hazard ratio. 
Table I. Association of the expression level of CD44 mRNA with clinicopathological factors of low-grade glioma and glioblastoma multiforme.

\begin{tabular}{|c|c|c|c|c|}
\hline \multirow[b]{2}{*}{ Clinicopathological feature } & \multirow[b]{2}{*}{ Patients, $n$} & \multicolumn{2}{|c|}{ CD44 mRNA expression } & \multirow[b]{2}{*}{ P-value } \\
\hline & & Low, $\mathrm{n}$ & High, $n$ & \\
\hline Age & & & & 0.126 \\
\hline$>50$ & 24 & 15 & 9 & \\
\hline$\leq 50$ & 29 & 12 & 17 & \\
\hline Sex & & & & 0.659 \\
\hline Male & 31 & 15 & 16 & \\
\hline Female & 22 & 12 & 10 & \\
\hline World Health Organization grade & & & & $<0.01$ \\
\hline II & 19 & 14 & 5 & \\
\hline III-IV & 34 & 13 & 21 & \\
\hline Karnofsky performance status & & & & $<0.01$ \\
\hline$>80$ & 19 & 15 & 4 & \\
\hline$\leq 80$ & 34 & 12 & 22 & \\
\hline
\end{tabular}

Table II. Univariate and multivariate analyses of the prognostic parameters of patients with low-grade glioma.

\begin{tabular}{lccccccc}
\hline & \multicolumn{3}{c}{ Univariate analysis } & & \multicolumn{3}{c}{ Multivariate analysis } \\
\cline { 2 - 3 } Variable & HR & $95 \%$ CI & P-value & & HR & $95 \%$ CI & P-value \\
\hline Age (>50 vs. $\leq 50)$ & 2.932 & $0.679-5.214$ & 0.127 & & 1.515 & $0.212-2.534$ & 0.099 \\
Sex (male vs. female) & 0.501 & $0.038-3.011$ & 0.450 & & 3.715 & $0.611-8.425$ & 0.679 \\
Karnofsky performance status (>80 vs. $\leq 80)$ & 0.246 & $0.001-1.091$ & 0.014 & & 0.417 & $0.046-1.914$ & 0.034 \\
CD44 mRNA expression (low vs. high) & 3.7012 & $0.927-11.215$ & 0.032 & & 2.862 & $0.436-5.534$ & 0.042
\end{tabular}

HR, hazard ratio.

in patients with high-grade glioma compared with patients with LGG and controls. Similar results were obtained using an mRNA PCR array and Oncomine analysis. However, there was no significant difference between grade III and IV glioma.

CD44 primarily regulates cell-to-cell and cell-to-extracellular matrix adhesion to preserve the organizational structure of tissues and organ. Additionally, CD44 is involved in cell migration, signaling, proliferation, angiogenesis, lymphocyte function and metastasis (19). In glioma, CD44 serves as an oncogene, and can promote GBM cell migration and invasion by influencing the mitogen-activated protein kinase/EPH receptor B2, epidermal growth factor and protein kinase B signaling pathways (20-22). Xu et al (23) demonstrated that CD44 is upregulated in GBM and promoted GBM cell growth and increased resistance to reactive oxygen speciesand cytotoxic agent-induced stress by inhibiting the Hippo signaling pathway. Although CD44 expression was positively correlated with malignancy in GBM, higher levels of CD44 were additionally correlated with decreased survival rates in these patients (24). However, the present study showed no statistically significant association between CD44 expression and OS in patients with GBM. The reason may be that the tumorigenicity of primary GBM differs between CD $44^{\text {low }} / \mathrm{CD} 133^{\text {high }}$ and CD $44^{\text {high }} / \mathrm{CD} 133^{\text {low }}$ for gene expression profiles (25). Furthermore, molecular heterogeneity among tumors affects the prognostic value of CD44 in GBM (26).

CD44 is highly upregulated in prostate, lung and pancreatic cancer, and is associated with poor prognosis (27-29). Nevertheless, the expression pattern and functional role of CD44 in LGG has not been fully elucidated. The present study identified that a high expression level of CD44 in patients with LGG were significantly associated with poor OS, and Kaplan-Meier analysis demonstrated that the expression level of CD44 may be used as a prognostic marker for patients. A recent study revealed that cells with low expression level of CD44 exhibited more glioma stem cell (GSC) traits, suggesting that CD44 is not an appropriate marker for GSCs, but CD44 can be used for predicting glioma-associated invasion and migration (30).

In conclusion, CD44 expression levels were associated with the histopathological grade of gliomas. Higher expression levels of CD44 mRNA were found in LGG and GBM tissues compared with normal brain tissues. A high expression level of CD44 mRNA was identified to be associated with a poor survival rate in LGG. The present results suggested that CD44 may be a novel 
prognostic biomarker that may improve OS prediction in LGG and serve as a potential therapeutic target for glioma. However, a larger sample size is required to further investigate the present results. Further experimental studies are required to elucidate the functions of CD44 in the progression of glioma.

\section{Acknowledgements}

Not applicable.

\section{Funding}

This project was supported by the Natural Science Foundation of Gansu (grant nos. 18JR3RA365 and 18JR3RA309), the Research Fund from The Project of the Health and Family Planning Commission of Gansu (grant no. GSWSKY-2014-31/ 2015-58), the Lanzhou Science and Technology Bureau Project (grantno.2018-1-109), and the DoctoralResearchFund andCuiying Science and Technology fund of Lanzhou University Second Hospital (grant nos. YNBSKYJJ2015-1-02/2015-2-11/2015-2-5 and CY2017-MS12/-MS15/CYXZ-01).

\section{Availability of data and materials}

The datasets used and/or analyzed during the present study are available from the corresponding author on reasonable request. The datasets generated and/or analyzed during the current study are available in the OncoLnc (http://www.oncolnc.org/search_ results/?q=CD44) and GEPIA (http://gepia.cancer-pku. cn/detail.php?gene $=\&$ clicktag $=$ survival) databases.

\section{Authors' contributions}

YP, GY and QD conceived the project. QD, QL, MW and JH performed the experiments. QD, QL and GY analyzed the data. QL, JH, LN and JD interpreted the data and revised the manuscript. YP, GY, QL and QD wrote the manuscript. All authors read and approved the final manuscript.

\section{Ethics approval and consent to participate}

The study was approved by The Ethical Committee of The Second Hospital of The Lanzhou University (Gansu, China). Written informed consent was provided by each patient or their guardians prior to their participation in the study.

\section{Patient consent for publication}

Not applicable.

\section{Competing interests}

The authors declare that they have no competing interests.

\section{References}

1. Louis DN, Perry A, Reifenberger G, von Deimling A, Figarellabranger D, Cavenee WK, Ohgaki H, Wiestler OD, Kleihues P and Ellison DW: The 2016 World Health Organization Classification of tumors of the central nervous system: A summary. Acta Neuropathol 131: 803-820, 2016.
2. Ostrom QT, Gittleman H, Xu J, Kromer C, Wolinsky Y, Kruchko C and Barnholtz-Sloan JS: CBTRUS statistical report: Primary brain and other central nervous system tumors diagnosed in the United States in 2009-2013. Neuro Oncol 18 (Suppl_5): v1-v75, 2016.

3. van den Bent MJ: Practice changing mature results of RTOG study 9802: Another positive PCV trial makes adjuvant chemotherapy part of standard of care in low-grade glioma. Neuro Oncol 16: 1570-1574, 2014.

4. Behin A, Hoangxuan K, Carpentier AF and Delattre JY: Primary brain tumours in adults. Lancet 379: 1984-1996, 2012.

5. Roszkowski K, Furtak J, Zurawski B, Szylberg T and Lewandowska M: Potential role of methylation marker in glioma supporting clinical decisions. Int J Mol Sci 17: 1876, 2016.

6. Yuan G, Niu L, Zhang Y, Wang X, Ma K, Yin H, Dai J, Zhou W and Pan Y: Defining optimal cutoff value of MGMT promoter methylation by ROC analysis for clinical setting in glioblastoma patients. J Neurooncol 133: 193-201, 2017.

7. Houillier C, Wang $\mathrm{X}$, Kaloshi G, Mokhtari $\mathrm{K}$, Guillevin R, Laffaire J, Paris S, Boisselier B, Idbaih A, Laigle-Donadey F, et al: IDH1 or IDH2 mutations predict longer survival and response to temozolomide in low-grade gliomas. Neurology 75: 1560-1566, 2010.

8. Felsberg J, Wolter M, Seul H, Friedensdorf B, Göppert M, Sabel MC and Reifenberger G: Rapid and sensitive assessment of the IDH1 and IDH2 mutation status in cerebral gliomas based on DNA pyrosequencing. Acta Neuropathol 119: 501-507, 2010.

9. Delgado-López PD and Corrales-García EM: Survival in glioblastoma: A review on the impact of treatment modalities. Clin Transl Oncol 18: 1062-1071, 2016.

10. Phan K, Ng W, Lu VM, McDonald KL, Fairhall J, Reddy R and Wilson P: Association between IDH1 and IDH2 mutations and preoperative seizures in patients with low-grade versus high-grade glioma: A systematic review and meta-analysis. World Neurosurg 111: e539-e545, 2018.

11. Sun L, Hui AM, Su Q, Vortmeyer A, Kotliarov Y, Pastorino S, Passaniti A, Menon J, Walling J, Bailey R, et al: Neuronal and glioma-derived stem cell factor induces angiogenesis within the brain. Cancer Cell 9: 287-300, 2006.

12. Chambless LB, Kistka HM, Parker SL, Hassam-Malani L, Mcgirt MJ and Thompson RC: The relative value of postoperative versus preoperative Karnofsky Performance Scale scores as a predictor of survival after surgical resection of glioblastoma multiforme. J Neurooncol 121: 359-364, 2015.

13. Li G, Shen J, Cao J, Zhou G, Lei T, Sun Y, Gao H, Ding Y, $\mathrm{Xu} \mathrm{W}$, Zhan Z, et al: Alternative splicing of human telomerase reverse transcriptase in gliomas and its modulation mediated by CX-5461. J Exp Clin Cancer Res 37: 78, 2018.

14. Takano S, Ishikawa E, Sakamoto N, Matsuda M, Akutsu H, Noguchi M, Kato Y, Yamamoto $T$ and Matsumura $A$ : Immunohistochemistry on IDH 1/2, ATRX, p53 and Ki-67 substitute molecular genetic testing and predict patient prognosis in grade III adult diffuse gliomas. Brain Tumor Pathol 33: 107-116, 2016.

15. Gulluoglu S, Tuysuz EC and Sahin M: Simultaneous miRNA and mRNA transcriptome profiling of glioblastoma samples reveals a novel set of OncomiR candidates and their target genes. Brain Res 1700: 199-210, 2018.

16. Valder CR, Liu JJ, Song YH and Luo ZD: Coupling gene chip analyses and rat genetic variances in identifying potential target genes that may contribute to neuropathic allodynia development. J Neurochem 87: 560-573, 2003.

17. Parkinson H, Sarkans U, Kolesnikov N, Abeygunawardena N, Burdett T, Dylag M, Emam I, Farne A, Hastings E, Holloway E, et al: ArrayExpress update-an archive of microarray and high-throughput sequencing-based functional genomics experiments. Nucleic Acids Res 39 (Database Issue): D1002-D1004, 2011.

18. Sedlazeck FJ, Lee H, Darby CA and Schatz MC: Piercing the dark matter: Bioinformatics of long-range sequencing and mapping. Nat Rev Genet 19: 329-346, 2018.

19. Dalal S, Zha Q, Daniels CR, Steagall RJ, Joyner WL, Gadeau AP, Singh $\mathrm{M}$ and Singh K: Osteopontin stimulates apoptosis in adult cardiac myocytes via the involvement of CD44 receptors, mitochondrial death pathway, and endoplasmic reticulum stress. Am J Physiol Heart Circ Physiol 306: H1182-H1191, 2014.

20. Feng C, Zhang Y, Yin J,Li J, Abounader R and Zuo Z: Regulatory factor $\mathrm{X} 1$ is a new tumor suppressive transcription factor that acts via direct downregulation of CD44 in glioblastoma. Neuro Oncol 16: 1078-1085, 2014. 
21. Monaghan M, Mulligan KA, Gillespie H, Trimble A, Winter P, Johnston PG and McCormick D: Epidermal growth factor up-regulates CD44-dependent astrocytoma invasion in vitro. J Pathol 192: 519-525, 2015.

22. Zhao LH, Lin QL, Wei J, Huai YL, Wang KJ and Yan HY: CD44v6 expression in patients with stage II or stage III sporadic colorectal cancer is superior to CD44 expression for predicting progression. Int J Clin Exp Pathol 8: 692-701, 2015.

23. Xu Y, Stamenkovic I and Yu Q: CD44 attenuates activation of the Hippo signaling pathway and is a prime therapeutic target for glioblastoma. Cancer Res 70: 2455-2464, 2010.

24. Wei KC, Huang CY, Chen PY, Feng LY, Wu TW, Chen SM, Tsai HC, Lu YJ, Tsang NM, Tseng CK, et al: Evaluation of the prognostic value of CD44 in glioblastoma multiforme. Anticancer Res 30: 253-259, 2010.

25. Fu J, Yang QY, Sai K, Chen FR, Pang JC, Ng HK, Kwan AL and Chen ZP: TGM2 inhibition attenuates ID1 expression in CD44-high glioma-initiating cells. Neuro Oncol 15: 1353-1365, 2013.

26. Nishikawa M, Inoue A, Ohnishi T, Kohno S, Ohue S, Matsumoto S, Suehiro S, Yamashita D, Ozaki S, Watanabe H, et al: Significance of Glioma Stem-Like cells in the tumor periphery that express high levels of CD44 in tumor invasion, early progression, and poor prognosis in glioblastoma. Stem Cells Int 2018: 5387041, 2018
27. Zeng Y, Wodzenski D, Gao D, Shiraishi T, Terada N, Li Y, Vander Griend DJ, Luo J, Kong C, Getzenberg RH and Kulkarni P: Stress-response protein RBM3 attenuates the stem-like properties of prostate cancer cells by interfering with CD44 variant splicing. Cancer Res 73: 4123-4133, 2013.

28. Guo JY, Hsu HS, Tyan SW, Li FY, Shew JY, Lee WH and Chen JY: Serglycin in tumor microenvironment promotes non-small cell lung cancer aggressiveness in a CD44-dependent manner. Oncogene 36: 2457-2471, 2017.

29. Zhao S, Chen C, Chang K, Karnad A, Jagirdar J, Kumar AP and Freeman JW: CD44 expression level and isoform contributes to pancreatic cancer cell plasticity, invasiveness and response to therapy. Clin Cancer Res 22: 5592-5604, 2016.

30. Wang HH, Liao CC, Chow NH, Huang LL, Chuang JI, Wei KC and Shin JW: Whether CD44 is an applicable marker for glioma stem cells. Am J Transl Res 9: 4785-4806, 2017.

(i) (i) () This work is licensed under a Creative Commons

C. AO ND Atribution-NonCommercial-NoDerivatives 4.0 International (CC BY-NC-ND 4.0) License. 